\title{
Ermittlung von Leistungsgrenzen verschiedener Lagerstrategien unter Berücksichtigung zentraler Einflussgrößen
}

\author{
Dipl.-Wirt.-Ing. (FH) Anne Piepenburg, Prof. Dr.-Ing. Rainer Bruns \\ Helmut-Schmidt-Universität, Hamburg \\ Lehrstuhl für Maschinenelemente und Technische Logistik
}

\begin{abstract}
Am Lehrstuhl für Maschinenelemente und Technische Logistik der HelmutSchmidt-Universität wurde ein neuer Modellierungsansatz zur Leistungsberechnung von Lagersystemen entwickelt. Das Modell basiert auf der mathematisch analytischen Berechnung der mittleren Spielzeit und ermöglicht die Berücksichtigung weitgehend beliebiger Lagerstrategien. Bei der Modellentwicklung wurden der lokale Auslastungsgrad sowie die lokale Verweildauer der einzelnen Lagerfächer als zentrale Einflussgrößen auf die Systemleistung identifiziert.
\end{abstract}

Das Modell ermöglicht erstmals die Einflüsse dieser beiden grundlegenden Größen analytisch zu beschreiben und damit tiefere Einsichten in die wesentlichen Wirkzusammenhänge zu gewinnen.

\section{$1 \quad$ Einleitung}

Automatisierte Lagersysteme verursachen hohe Kosten durch den Lagerbetrieb aber auch durch die Abschreibung der Investition. Deshalb wird seit langem versucht die logistische Systemleistung nicht nur durch eine Steigerung der Leistungsfähigkeit der eingesetzten Technik, sondern insbesondere durch ausgefeilte Lagerstrategien d.h. durch eine intelligente Zuordnung der Lagereinheiten zu den Lagerfächern zu erhöhen. Die Auswahl der für den jeweiligen Anwendungsfall richtigen Lagerstrategie ist einerseits von großer Bedeutung für die Wirtschaftlichkeit des Lagersystems und andererseits keinesfalls trivial. Voraussetzung hierfür ist die Möglichkeit, verschiedene Lagerstrategien objektiv vergleichen und bewerten zu können. Dieses kann mithilfe von Computersimulationen unter definierten Einsatzszenarien geschehen. Tiefere Einsichten in die wesentlichen Zusammenhänge zwischen den Einflussparametern und der Zielgröße Umschlagsleistung lassen sich dabei jedoch nur schwer gewinnen. Das in diesem Zusammenhang entwickelte Modell soll im folgenden Beitrag vorgestellt werden.

\section{Grundlagen zum Lager}

\subsection{Stochastische Einflussgrößen im Lager}

Einige der in einem Lager auftretenden Parameter können durch Wahrscheinlichkeitsverteilungen beschrieben werden. Im vorliegenden Beitrag sind das insbesondere die Fahrzeitverteilung und die Verweildauerverteilung. 
Die Dichtefunktion der Fahrzeitverteilung gibt die Wahrscheinlichkeit an, mit welcher eine bestimmte Spielzeit $t_{s}$ bei einem Einzelspiel eintritt. Die Wahrscheinlichkeit nimmt bei chaotischer Lagerung mit steigendem $t_{s}$ linear $z u$ (vgl. Abb. 1), weil die Anzahl von Lagerfächern mit gleicher Spielzeit mit zunehmendem Abstand vom Übergabepunkt wächst. Voraussetzung hierfür ist, dass das Verhältnis von Lagerhöhe zu Lagerbreite dem Verhältnis von Hubgeschwindigkeit zu Fahrgeschwindigkeit gleicht.

Die Verweildauer eines Artikels ist bei bestimmten Lagerstrategien Entscheidungsgrundlage, in welches Lagerfach (LF) der Artikel eingelagert wird. Da die Verweildauer des Artikels jedoch zum Einlagerungszeitpunkt unbekannt ist, liegt die Verweildauerverteilung idealerweise aufgrund von Erfahrungswerten vor. Die Verweildauerverteilung gibt an, mit welcher Wahrscheinlichkeit eine bestimmte Verweildauer eintritt, sodass - abhängig von der verwendeten Lagerstrategie - der Einlagerungsort für die Lagereinheit (LE) bestimmt werden kann. Die Interpretation der Verweildauerverteilung soll im Folgenden anhand einer gleichverteilten Verweildauer erfolgen.

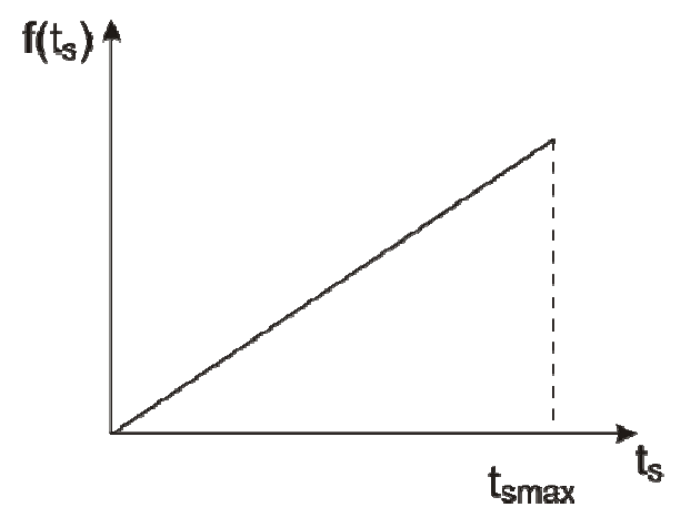

Abbildung 1: Dichtefunktion der Fahrzeitverteilung

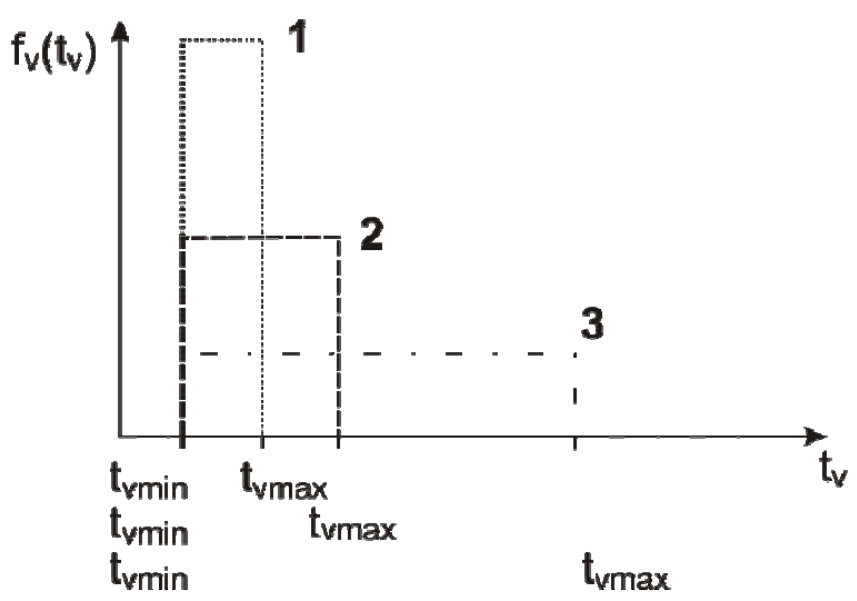

Abbildung 2: Unterschiedliche Gleichverteilungen der Verweildauer

Wenn eine Zufallsgröße (in diesem Fall die Verweildauer) gleichverteilt ist, besteht für jede mögliche Verweildauer die gleiche Eintrittswahrscheinlichkeit. Abbildung 2 zeigt drei Dichtefunktionen einer Gleichverteilung. Liegt eine Gleichverteilung wie bei 1 vor, so unterscheiden sich die Verweildauern im Sortiment kaum. Bei stark unterschiedlichen Verweildauern hat die Dichtefunktion der Gleichverteilung den Verlauf 3.

\subsection{Lagerstrategien}

Als Lagerstrategien werden Verfahren bezeichnet, nach denen ein Lagerfach für die Einlagerung ausgewählt wird. Gängige Lagerstrategien sind beispielsweise die chaotische Lagerung, die Kürzeste-Fahrzeit-Strategie oder die ABC-Zonierung. Bei der chaotischen Lagerung werden ankommende Ladeeinheiten zufällig Lagerfächern 
zugewiesen, sodass für jedes Lagerfach die gleiche Wahrscheinlichkeit besteht, angefahren zu werden. Die Kürzeste-Fahrzeit-Strategie (KFZ) hingegen wählt unabhängig vom einzulagernden Artikel immer den Lagerort mit dem kürzesten Fahrweg aus. Wird das Lager in Zonen eingeteilt, spricht man oftmals von einer ABC-Zonierung. Innerhalb einer Zone werden nur Ladeeinheiten mit definierten Attributsausprägungen eingelagert [tHo08]. Eine weitere Lagerstrategie, die in der Praxis bislang kaum Anwendung findet, ist die dynamische Zonierung. Die dynamische Zonierung wurde von Michael Glass entwickelt. Glass geht davon aus, dass die Verweildauerverteilung der Artikel sowie die Fahrzeitverteilung im Lager vor Einlagerung bekannt sind. Um den Lagerort eines ankommenden Artikels zu bestimmen wird dessen Verweildauerquantil mit dem Quantil in der Fahrzeitverteilung gleichgesetzt. Über die Inverse der Fahrzeitverteilungsfunktion lässt sich schließlich die Fahrzeit zu dem gesuchten Lagerort für den Artikel berechnen (vgl. Abb. 3 und Gl. 2.1) [Gla08]:

$t_{F}=F_{F}^{-1}\left(F_{V}\left(t_{V}\right)\right)$
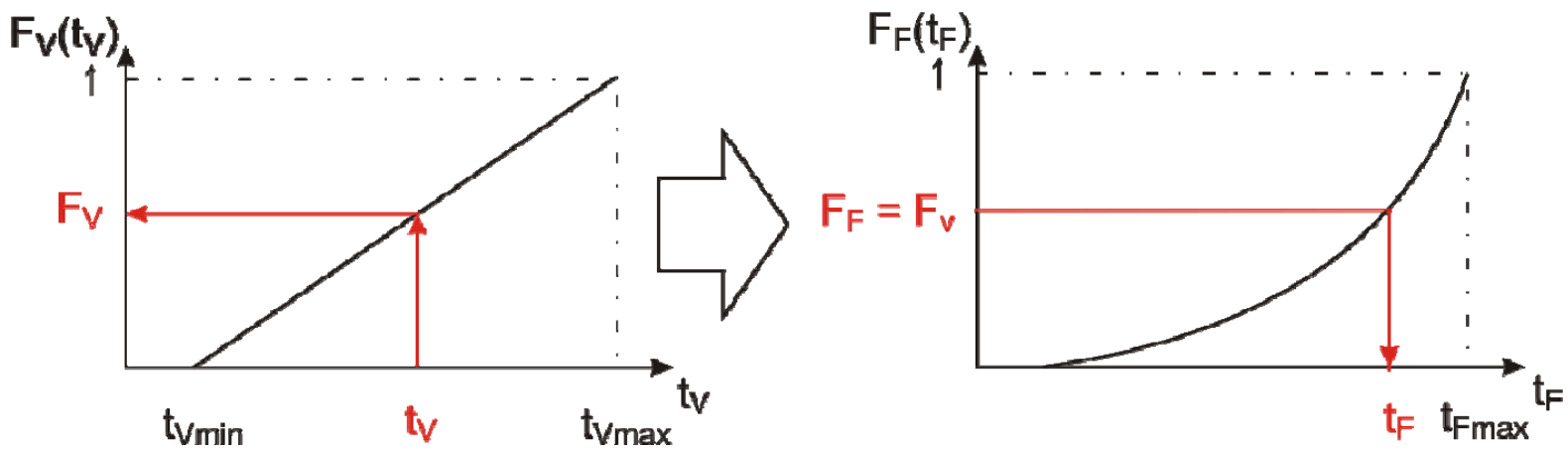

Abbildung 3: Verweildauerverteilung des Artikels (links) und die Fahrzeitverteilung des Lagers (rechts)

In dem vorliegenden Beitrag finden die chaotische Lagerung und die dynamische Zonierung nach Glass Anwendung.

\section{$3 \quad$ Modell zur Leistungsberechnung}

\subsection{Voraussetzung und Annahmen zur Modellbildung}

Die Leistung eines Lagers wird durch die mittlere Spielzeit charakterisiert. Die Spielzeit eines Lagerfaches ist die Zeitdauer, die für die Ausführung eines Arbeitsspiels, d.h. einer Aus- oder Einlagerung benötigt wird. Das Arbeitsspiel ist dabei eine zyklische Wiederholung von einzelnen Arbeitsoperationen. Werden die für die Arbeitsoperationen benötigten Zeitanteile addiert, ergibt sich die Spielzeit für ein bestimmtes Arbeitsspiel. Diese Zeitanteile werden in wegabhängige und wegunabhängige Zeitanteile unterschieden. Die Spielzeit hängt im Wesentlichen von der Länge des beim Transportvorgang zurückzulegenden Weges ab. Somit ist die Spielzeit für jedes Lagerfach unterschiedlich, sie wird als spezielle Spielzeit bezeichnet [Bru09]. 
In dem entwickelten Modell können zeitliche Schwankungen nicht betrachtet werden. Bei den in dem Modell verwendeten Größen handelt es sich um zeitliche Mittelwerte, sodass nur stationäre Zustände durch das Modell beschrieben werden können.

Zunächst wird nur eine einzelne Regalfläche modelliert. Das Modell kann jedoch auf mehrere Regalflächen erweitert werden. Die modellierte Regalfläche besteht aus $\mathrm{M} \mathrm{x}$ $\mathrm{N}$ gleichgroßen Lagerfächern (vgl. Abb. 4).

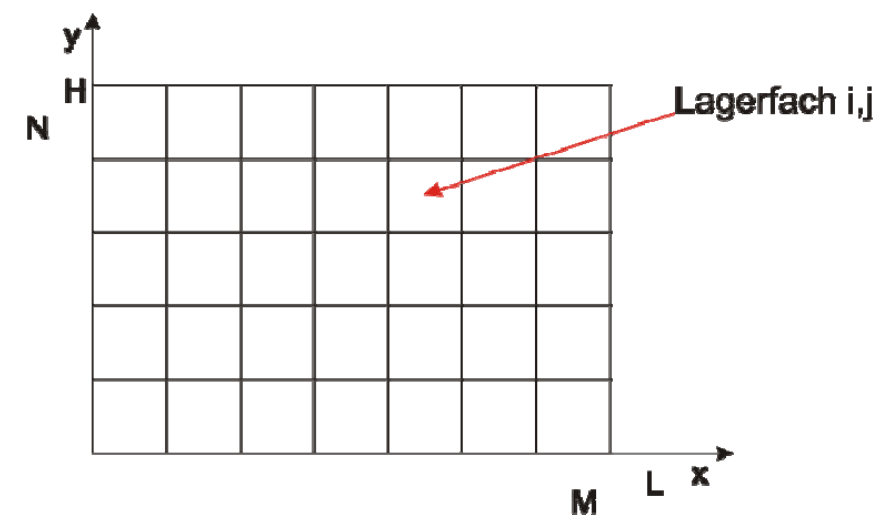

Abbildung 4: modellierte Regalfläche

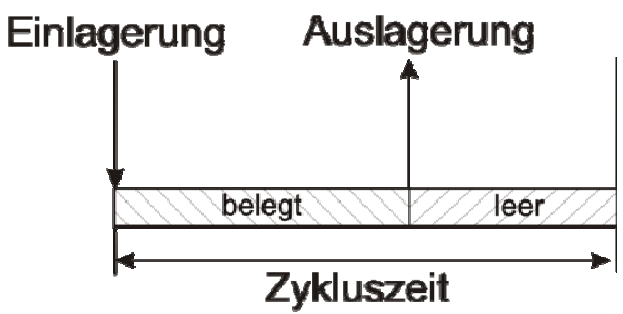

Abbildung 5: Zykluszeit eines Lagerfachs

Jedes Lagerfach ist eindeutig durch seine Indizes i, j gekennzeichnet. Für jedes Lagerfach sei die Spielzeit $t_{\mathrm{sij}}$, die Verweildauer $t_{\mathrm{vij}}$ und der Auslastungsgrad $\rho_{\mathrm{ij}}$ bekannt.

Der Auslastungsgrad $\rho_{\mathrm{ij}}$ beschreibt das Verhältnis der Zwischenankunftszeit $t_{\mathrm{Aij}}$ (Zykluszeit) und der Verweildauer $\mathrm{t}_{\mathrm{vij}}$

$\rho_{i j}=\frac{t_{v i j}}{t_{A i j}}$

Die Zwischenankunftszeit setzt sich dabei aus der Verweildauer einer Lagereinheit und der Zeitdauer $t_{\text {leerij, }}$ in der das Lagerfach leer ist, zusammen. Abbildung 5 zeigt den typischen Zyklus, den jedes Lagerfach durchläuft. Die Zeitanteile zwischen Einlagerung und Auslagerung sowie die Leerzeit können bei jedem Zyklus variieren. Es gilt also:

$t_{\text {Aij }}=t_{\text {vij }}+t_{\text {leerij }}$

Hieraus folgt durch Umstellen von Gl. 3.1 und anschließendes Einsetzen in GI. 3.2:

$t_{A i j}=\frac{t_{v i j}}{\rho_{i j}}$ und $\quad t_{l e e r i j}=\frac{1-\rho_{i j}}{\rho_{i j}} \cdot t_{v i j}$

Um die Anfahrwahrscheinlichkeit $P_{i j}$ eines Lagerfaches ij zu bestimmen, wird die Regalfläche als eine Parallelschaltung vieler (genauer $M \times N$ ) Bedienstationen aufgefasst (vgl. Abb. 6). 


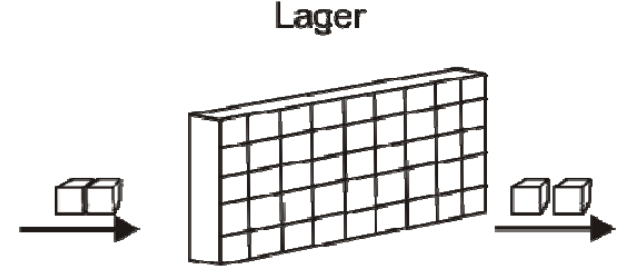

Eingangsdurchsatz $\lambda_{\text {in }}$
Ausgangs-

durchsatz $\lambda_{\text {out }}$

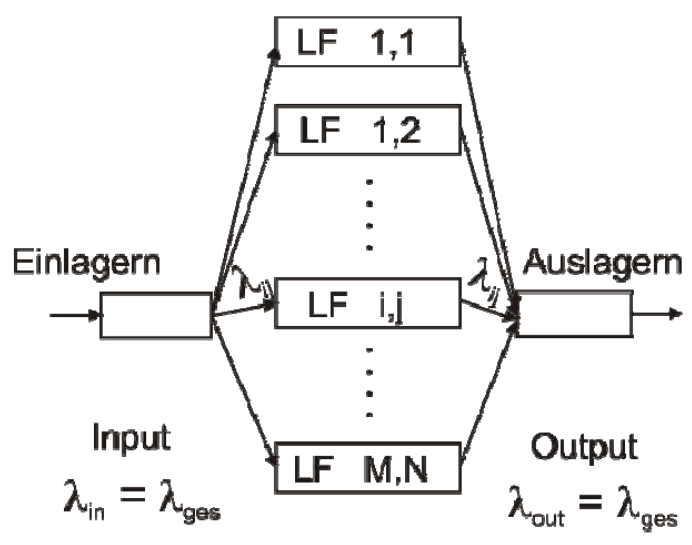

Abbildung 6: Regalfläche als Bedienstation

Die Wahrscheinlichkeit, dass eine ankommende LE ins Lagerfach LF $_{i j}$ eingelagert wird, ergibt sich aus dem relativen Durchsatz $\frac{\lambda_{i j}}{\lambda_{\text {ges }}}$ des Lagerfaches.

Es gilt also:

$P_{i j}=\frac{\lambda_{i j}}{\lambda_{\text {ges }}}$

Der zeitlich gemittelte Durchsatz $\lambda_{\mathrm{ij}}$ ist der Kehrwert der Zwischenankunftszeit $t_{\mathrm{Aij}}$ des Lagerfaches

$\lambda_{i j}=\frac{1}{t_{A i j}}$

Durch das Aufsummieren aller Einzeldurchsätze erhält man den Gesamtdurchsatz durch die gesamte Lagerfläche

$\lambda_{\text {ges }}=\sum_{i} \sum_{j} \lambda_{i j}=\lambda_{z u}=\lambda_{a b}$

Der örtlich gemittelte Durchsatz $\lambda_{m}$ ergibt sich, wenn man den Gesamtdurchsatz auf die Anzahl M x N der Lagerfächer in der Lagerfläche bezieht.

$\lambda_{m}=E\left(\lambda_{i j}\right)=\frac{\lambda_{g e s}}{M \cdot N}$

\subsection{Leistungsberechnung mit einem diskreten Modell}

Die mittlere Spielzeit $t_{\mathrm{sm}}$ ist ein Mittelwert für die beim Einzelspiel benötigte Zeitdauer. Sie ist der Erwartungswert der speziellen Spielzeiten für die einzelnen Lagerfächer:

$t_{s m}=\sum_{j} \sum_{i} P_{i j} \cdot t_{s i j}$

Mit Gleichung 3.5 folgt:

(c) 2011 Logistics Journal : Proceedings - ISSN 2192-9084 
$t_{s m}=\sum_{i=1}^{M} \sum_{j=1}^{N} \frac{\lambda_{i j}}{\lambda_{g e s}} \cdot t_{s i j}$

Mit Gleichung 3.8 folgt weiter:

$t_{s m}=\frac{1}{M N} \cdot \sum_{i=1}^{M} \sum_{j=1}^{N} \frac{\lambda_{i j}}{\lambda_{m}} \cdot t_{s i j}$

Der Quotient $\frac{\lambda_{i j}}{\lambda_{m}}$ kann als lagerfachabhängiger Einflussfaktor gedeutet werden.

Durch Rückführung der Lagerfachdurchsätze $\lambda_{\mathrm{ij}}$ auf die Ursprungsgrößen $\mathrm{t}_{\mathrm{vij}}$ und $\rho_{\mathrm{ij}}$ (vgl. Gl. 3.3 und 3.6) erhält man:

$t_{s m}=\frac{\sum_{i=1}^{M} \sum_{j=1}^{N} \frac{\rho_{i j}}{t_{v i j}} t_{s i j}}{\sum_{i=1}^{M} \sum_{j=1}^{N} \frac{\rho_{i j}}{t_{v i j}}}$

\subsection{Leistungsberechnung mit einem stetigen Modell}

Durch den Grenzübergang zu unendlich vielen Lagerfächern $(N \rightarrow \infty, M \rightarrow \infty)$ kann eine Kontinuierung des Problems durchgeführt werden. Somit werden die Lagerfächer bei gleichbleibender Regalfläche infinitesimal klein. Unter der Annahme, dass alle Lagerfächer gleich groß sind, ergibt sich die Breite eines Lagerfaches aus der Länge $L$ dividiert durch die Anzahl $M$ der Lagerfächer in $x$ - Richtung:

$\Delta x=\frac{L}{M}, \quad \lim _{M \rightarrow \infty} \Delta x=d x$

Entsprechendes gilt für die Höhe eines Lagerfaches:

$$
\begin{aligned}
& \Delta y=\frac{H}{N}, \quad \lim _{N \rightarrow \infty} \Delta y=d y \\
& \rightarrow \frac{1}{M N}=\frac{\Delta x}{L} \frac{\Delta y}{H}
\end{aligned}
$$

Durch Einsetzen in (3.10) und Kontinuierung von $\lambda_{\mathrm{ij}}$ und $\mathrm{t}_{\mathrm{sij}}$ erhält man:

$\lambda_{i j} \rightarrow \lambda(x, y), t_{s i j} \rightarrow t_{s}(x, y)$

$t_{s m}=\frac{1}{L H} \iint_{x=0 y=0}^{L H} \frac{\lambda(x, y)}{\lambda_{m}} \cdot t_{s}(x, y) d x d y$

aus (3.12) folgt schließlich: 
$t_{S}=\frac{\iint_{x=0 y=0}^{L H} \frac{\rho(x, y)}{t_{v}(x, y)} t_{S}(x, y) d x d y}{\iint_{x=0 y=0}^{L H} \frac{\rho(x, y)}{t_{v}(x, y)} d x d y}$

mit $\rho_{i j} \rightarrow \rho(x, y), t_{v i j} \rightarrow t_{v}(x, y)$

\subsection{Leistungsberechnung mit einem klassiert diskreten Modell}

In jedem Lager gibt es Klassen von Lagerfächern die hinsichtlich der relevanten Größen gleiche Werte aufweisen. Diese Fächer liegen auf einer gemeinsamen Linie, der sogenannten Isolinie. Eine Isolinie verbindet alle Punkte (bzw. LF), für die man von einem bestimmten Punkt aus (z.B. der Übergabepunkt in einem Lager) die gleiche spezielle Einzelspielzeit benötigt [Arn09]. Es werden daher $M_{k}$ Teilmengen $K_{k}$ von Lagerfächern gebildet. Für alle Elemente einer Klasse sind die Werte der Auslastung $\rho_{i j}$, der Verweildauer $t_{v i j}$ und der Spielzeit $t_{s i j}$ gleich groß.

Es gilt:

$$
\begin{aligned}
& \rho_{i j}=\rho_{k} \\
& t_{v i j}=t_{v k} \\
& t_{s i j}=t_{s k}
\end{aligned}
$$

$$
\begin{aligned}
& \forall k \in\left[1, M_{k}\right] \wedge \\
& \forall i, j \in K_{k}
\end{aligned}
$$

Die Anzahl der Lagerfächer (Elemente) der Klasse $K_{k}$ sei die Häufigkeit $h_{k}$. Die relative Häufigkeit der Klassen ist dann:

$$
h_{k, r e l}=\frac{h_{k}}{M N}
$$

Mit Gleichung (3.12) erhält man durch die Klassierung:

$$
t_{s m}=\frac{\sum_{k} h_{k, r e l} \frac{\rho_{k}}{t_{v k}} t_{s k}}{\sum_{k} h_{k, r e l} \frac{\rho_{k}}{t_{v k}}}
$$

\subsection{Leistungsberechnung mit einem klassiert kontinuierlichen Modell}

Nimmt man eine weitere kontinuierliche Verfeinerung der Klassierung bis zum Grenzfall $M_{k} \rightarrow \infty$ vor, so kann die Zuordnung der Klassen nicht mehr durch den Index $\mathrm{k}$ erfolgen. Hierfür wird ein stetiger Parameter benötigt. Da die Klassierung üblicher Weise anhand der Spielzeit $t_{s}$ erfolgt, bietet es sich an, die relevanten Parameter in Abhängigkeit von der Spielzeit $t_{s}$ darzustellen: 
$\rho_{k} \rightarrow \rho\left(t_{s}\right), t_{v k} \rightarrow t_{v}\left(t_{s}\right)$

Bei wachsendem $M_{k}$ strebt die Klassenhäufigkeit $h_{k}$ bzw. $h_{k, \text { rel }}$ gegen null. Diese muss daher auf die Klassenbreite $\Delta \mathrm{t}_{\mathrm{s}}$ bezogen werden:

$\lim _{M_{k} \rightarrow \infty} \frac{h_{k, r e l}}{\Delta t_{s}}=f_{s 0}\left(t_{s}\right)$

Im Grenzübergang $M_{k} \rightarrow \infty$ strebt der Quotient $\frac{h_{k, \text { rel }}}{\Delta t_{s}}$ gegen die Dichtefunktion der Spielzeitverteilung bei chaotischer Lagerung. Der Quotient wird im Folgenden als geometrischer Spielzeitfaktor bezeichnet.

Der geometrische Spielzeitfaktor lässt sich mit Hilfe der Dichtefunktion der Spielzeitverteilung (vgl. Abb. 7) bei chaotischer Lagerung bestimmen. Es gilt:

$f_{s 0}\left(t_{s}\right)=f_{s 0 \max } \cdot \frac{t_{s}-t_{s \min }}{t_{s \max }-t_{s \min }} \quad \forall t_{s} \in\left[t_{s \min }, t_{s \max }\right]$

$\mathrm{f}_{\mathrm{s} 0 \mathrm{max}}$ wird mit Hilfe der Bedingung bestimmt, dass die Fläche unter der Dichtefunktion 1 sein muss:

$$
\begin{aligned}
& \frac{1}{2} \cdot f_{s 0 \text { max }} \cdot\left(t_{s \max }-t_{s \min }\right)=1 \\
& \rightarrow f_{s 0 \text { max }}=\frac{2}{\left(t_{s \max }-t_{s \min }\right)}
\end{aligned}
$$

Damit ergibt sich für die Dichtefunktion:

$$
f_{s 0}\left(t_{s}\right)=\frac{2 \cdot\left(t_{s}-t_{s \min }\right)}{\left(t_{s \max }-t_{s \min }\right)^{2}}
$$

Für die weiteren Berechnungen mit dem Leistungsmodell wird eine Variablensubstitution mit der Hilfsvariablen $\tau$ durchgeführt. Die Substitution ermöglicht eine Integration im Wertebereich $0 \leq \tau \leq 1$. Die Dichtefunktion wird damit in den Ursprung verschoben und normiert (vgl. Abb. 7). Die Dichtefunktion in Abhängigkeit der Hilfsvariablen zeigt Gleichung 3.27. 


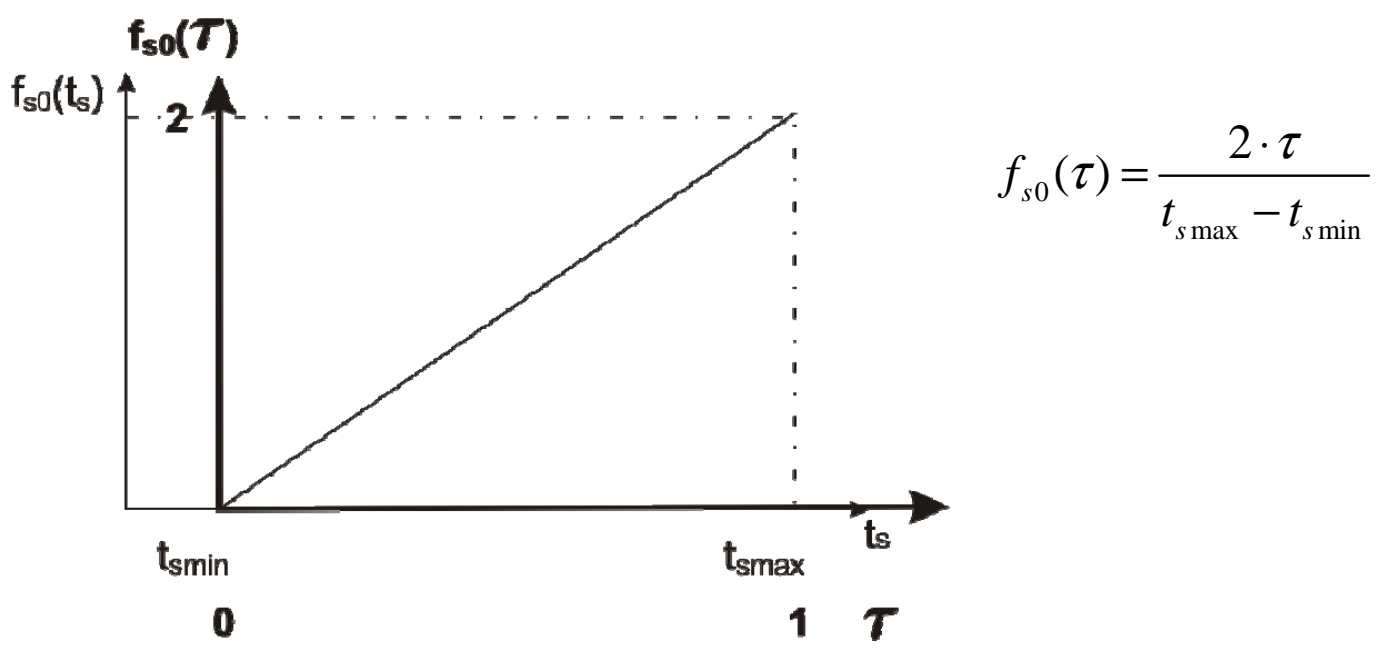

Abbildung 7: Geometrische Verteilung der Spielzeit

Mit den dargestellten Übergängen für $M_{k} \rightarrow \infty$ folgt aus der Bestimmungsgleichung (3.21):

$t_{s m}=\frac{\int_{t_{\text {min }}}^{t_{s \max }} f_{s 0}\left(t_{s}\right) \frac{\rho\left(t_{s}\right)}{t_{v}\left(t_{s}\right)} t_{s} d t_{s}}{\int_{t_{s \min }}^{t_{s \max }} f_{s 0}\left(t_{s}\right) \frac{\rho\left(t_{s}\right)}{t_{v}\left(t_{s}\right)} d t_{s}}$

$t_{s m i n}$ und $t_{s \max }$ sind die obere und untere Grenze des Wertebereichs der Spielzeit $t_{s}$. Nach der Variablensubstitution erhält man:

$$
t_{s m}=\frac{\int_{0}^{1} f_{s 0}(\tau) \frac{\rho(\tau)}{t_{v}(\tau)}\left(t_{s \min }+\Delta t_{s} \cdot \tau\right) d \tau}{\int_{0}^{1} f_{s 0}(\tau) \frac{\rho(\tau)}{t_{v}(\tau)} d \tau} \quad \text { mit } \tau=\frac{t_{s}-t_{s \min }}{t_{s \max }-t_{s \min }}
$$

\subsection{Modellvalidierung anhand der chaotischen Lagerung}

Die Modellvalidierung erfolgt anhand der chaotischen Lagerung. Für die Berechnung der mittleren Einzelspielzeit bei chaotischer Lagerung liegt eine analytische Formel vor, die die Spielzeit in Abhängigkeit der Bewegungsgrößen des Fördermittels bei parallelem Bewegungsablauf sowie konstanten Zeitanteilen für die Lastaufnahme und Lastabgabe und der Lagerausmaße bestimmt. Bei Gültigkeit des Modells zur Leistungsbestimmung muss das Ergebnis für die mittlere Spielzeit mit dem Ergebnis der bekannten analytischen Formel übereinstimmen. Die analytische Formel für die mittlere Einzelspielzeit bei chaotischer Lagerung lautet:

$t_{s m}=\frac{t_{K A}+t_{K E}}{2}+2 t_{0}+\frac{4}{3} \cdot \frac{L}{u}$

mit $t_{K A} \quad$ konstanter Zeitanteil für das Auslagern

(c) 2011 Logistics Journal : Proceedings - ISSN 2192-9084 
$t_{\mathrm{KE}} \quad$ konstanter Zeitanteil für das Einlagern

$2 t_{0} \quad$ Zeitanteil für $2 \times$ Beschleunigen und Abbremsen

L Lagerlänge

u Maximalgeschwindigkeit Fahren

Für die Berechnung nach dem Leistungsmodell werden zunächst die einzusetzenden Funktionsparameter bestimmt. Bei der Einlagerung nach chaotischer Strategie werden die Verweildauern des einzulagernden Artikels nicht berücksichtigt. Daher ist die Verweildauer im Mittel über die Regalfläche als konstant anzusehen. Gleiches gilt für die Auslastung, da diese bei der Einlagerung nach chaotischer Strategie nicht berücksichtigt wird. Nach Einsetzen in GI. (3.29) erhält man:

$t_{s m}=\frac{\int_{0}^{1} \frac{2 \tau}{t_{s \max }-t_{s \min }} \cdot \frac{\rho}{t_{v}} \cdot\left(t_{s \min }+\left(t_{s \max }-t_{s \min }\right) \cdot \tau\right) d \tau}{\int_{0}^{1} \frac{2 \tau}{t_{s \max }-t_{s \min }} \cdot \frac{\rho}{t_{v}} d \tau}$

$t_{s m}=t_{s \min }+\left(t_{s \max }-t_{s \min }\right) \cdot \frac{\int_{0}^{1} \tau^{2} d \tau}{\int_{0}^{1} \tau \cdot d \tau}$

Nach Lösen der beiden Integrale erhält man schließlich:

$t_{s m}=t_{s \min }+\frac{4}{3} \cdot \frac{L}{u}$

Der Zeitanteil $t_{s m i n}$ setzt sich aus dem konstanten Zeitanteil und den beiden Zeitanteilen für die Beschleunigung zusammen. Damit ist die Gültigkeit der Berechnung der mittleren Spielzeit nach dem Leistungsmodell für den Spezialfall der chaotischen Lagerung nachgewiesen.

\section{Leistungsberechnung für die dynamische Zonierung bei gleichverteilter Verweildauer}

Die Leistungsberechnung soll im Folgenden auf die dynamische Zonierung von Glass angewendet werden. Es wird eine gleichverteilte Verweildauer angenommen. Gemäß der dynamischen Zonierung werden die Quantile der Fahrzeit- und Verweildauerverteilungsfunktion gleichgesetzt. Es liegt die geometrische Spielzeitverteilung vor. Die erforderliche Fahrzeitverteilungsfunktion $F_{F}(\tau)$ entspricht der Verteilungsfunktion $F_{s 0}(\tau)$ des geometrischen Spielzeitfaktors $f_{s 0}(\tau)$. Die Verweildauer wird in Abhängigkeit von $\tau$ bestimmt.

Es gilt:

$F_{s 0}(\tau)=F_{v}\left(t_{v}\right)$

(c) 2011 Logistics Journal : Proceedings - ISSN 2192-9084 
$\Rightarrow t_{v}(\tau)=t_{v \text { min }}+\Delta t_{v} \cdot \tau^{2}$ mit $\Delta t_{v}=t_{v \text { max }}-t_{v \text { min }}$

Bei der Lagerstrategie "dynamische Zonierung" ist die Auslastung $\rho$ für alle Lagerfächer gleich groß, sodass $\rho$ als konstant angenommen werden kann. Es liegt der geometrische Spielzeitfaktor $f_{s 0}(\tau)$ vor. Die ermittelten Parameter werden anschließend in das Leistungsmodell (vgl. Gl. 3.29) eingesetzt. Nach Vereinfachung erhält man [Bro01]:

$$
\begin{gathered}
t_{s m}=t_{s \text { min }}+\Delta t_{s} \cdot \frac{\int_{0}^{1} \frac{\tau^{2} d \tau}{t_{v \text { min }}+\Delta t_{v} \cdot \tau^{2}}}{\int_{0}^{1} \frac{\tau d \tau}{t_{v \text { min }}+\Delta t_{v} \cdot \tau^{2}}} \\
t_{s m}=t_{s \text { min }}+\Delta t_{s} \cdot \frac{2 \cdot\left(1-\sqrt{\frac{t_{v \min }}{\Delta t_{v}}} \cdot \arctan \left(\sqrt{\frac{\Delta t_{v}}{t_{v \text { min }}}}\right)\right)}{\ln \frac{t_{v \text { max }}}{t_{v \text { min }}}}
\end{gathered}
$$

Die Gleichung für die mittlere Spielzeit setzt sich nun aus der Summe von $t_{s m i n}$ und $\Delta \mathrm{t}_{\mathrm{s}}$ zusammen, wobei $\Delta \mathrm{t}_{\mathrm{s}}$ mit einem Faktor multipliziert wird, der sich nach Lösen des Nenner und des Zählerintegrals und anschließender Division ergibt. Dieser Faktor wird im Folgenden als Einflussfaktor $\alpha$ bezeichnet. Er gibt die Leistungsfähigkeit einer Lagerstrategie an. Bei chaotischer Lagerung ist der Einflussfaktor gleich 2/3. Tabelle 1 zeigt die Ergebnisse für den Einflussfaktor bei Variation des Intervalls für die Verweildauer. Intervall 1 beschreibt den chaotischen Grenzfall, denn $t_{v}$ ist nahezu konstant, sodass nach der dynamischen Zonierung annähernd chaotisch eingelagert wird. Ein großes Intervall (vgl. Intervall 3) erwirkt einen niedrigen Einflussfaktor und damit eine kurze mittlere Spielzeit. Die Strategie "dynamische Zonierung" erzielt bei stark unterschiedlichen Verweildauern im Sortiment folglich die besten Ergebnisse.

\begin{tabular}{|l|l|l|l|l|}
\hline & $\mathbf{t}_{\mathrm{vmin}}$ & $\mathbf{t}_{\mathrm{vmax}}$ & $\Delta \mathbf{t}_{\mathrm{v}}$ & Einflussfaktor $\boldsymbol{\alpha}$ \\
\hline $\mathbf{1 .}$ & 1 & 1,1 & 0,1 & 0,66 \\
\hline $\mathbf{2 .}$ & 1 & 11 & 10 & 0,5 \\
\hline $\mathbf{3 .}$ & 1 & 1001 & 1000 & 0,275 \\
\hline
\end{tabular}

Tabelle 1: Ergebnisse für den Einflussfaktor

\section{$5 \quad$ Zusammenfassung und Ausblick}

Bei der Modellentwicklung hat sich gezeigt, dass die Leistungsberechnung von Lagerstrategien anhand weniger Parameter erfolgen kann. Dabei wurden der lokale Auslastungsgrad der einzelnen Lagerfächer sowie die Verweildauer der Lagereinheiten in den Lagerfächern als zentrale Einflussgrößen auf die 
Systemleistung identifiziert. Beide Größen werden durch die Lagerstrategie entscheidend beeinflusst.

Erste Ergebnisse der Leistungsberechnung machten das Optimierungspotenzial der dynamischen Zonierung gegenüber der chaotischen Lagerung deutlich und konstatierten den signifikanten Einfluss der Verweildauer auf die mittlere Spielzeit.

Mit der Einführung der dynamischen Zonierung wurde bereits ein Fortschritt in der Reduzierung der mittleren Spielzeit erzielt. In weiteren Schritten sollte die Auswirkung der Auslastung der Regalfläche auf die mittlere Spielzeit untersucht werden. Die Auslastung der Regalfläche fließt bislang nicht in die Strategie "dynamische Zonierung" ein, obwohl diese - nach heutigem Kenntnisstand - einen wesentlichen Einfluss auf die mittlere Spielzeit hat. Eine weitere Steigerung der Lagerleistung durch die Kombination der dynamischen Zonierung mit der KürzestenFahrzeit-Strategie ist infolgedessen zu erwarten.

\section{Literatur}

[Arn09] Arnold, D.; Furmans, K.: Materialfluss in Logistiksystemen. Berlin Heidelberg: Springer-Verlag, 2009

[Bro01] Bronstein, I.N. et al.: Taschenbuch der Mathematik. Frankfurt am Main: Verlag Harri Deutsch, 2001

[Bru09] Bruns, R.: vorlesungsbegleitendes Skript Logistik 2. Hamburg: HelmutSchmidt-Universität, 2009

[Gla08] Glass, M.: Schnelläuferstrategien in Lagern und Dynamische Zonierung. Doktorarbeit, Technische Universität Dresden, 2008

[tHo08] ten Hompel, M.; Schmidt, T.: Warehouse Management, Organisation und Steuerung von Lager- und Kommissioniersystemen, Berlin Heidelberg: Springer-Verlag, 2008 